\title{
Emergence and Polyclonal Dissemination of OXA-244- Producing Escherichia coli, France
}

\author{
Cecile Emeraud, Delphine Girlich, Rémy A. Bonnin, Agnès B. Jousset, Thierry Naas, Laurent Dortet
}

Since 2016, OXA-244-producing Escherichia coli has been increasingly isolated in France. We sequenced 97 OXA-244-producing E. coli isolates and found a wide diversity of sequence types and a high prevalence of sequence type 38. Long-read sequencing demonstrated the chromosomal location of bla ${ }_{\text {OXA-244 }}$ inside the entire or truncated $\operatorname{Tn} 51098$.

arbapenems are the last line antimicrobial drugs for treating infections caused by multidrug-resistant Enterobacterales. The global dissemination of carbapenemase-producing Enterobacterales (CPE; formerly known as carbapenemase-producing Enterobacteriaceae) pose a serious threat to public health (1). Oxacillin (OXA) 244, a single amino-acid variant of OXA-48 (Arg-222-Gly) (2), is an emerging carbapenemase variant in several countries in Europe (3-7). During 2013-2019, the French National Reference Center received a continuously increasing number of OXA-244-producing isolates for antimicrobial resistance (AMR) testing. OXA-244-producing isolates increased from 0 in 2012 to 72 in 2019. In France, OXA-244-producing Enterobacteriaceae represent $2.4 \%$ of all CPE and represented $3.4 \%$ of OXA-48-like producing CPE in 2019 (8). In addition, this tendency might represent only a fraction of OXA-244-producing Enterobacteriaceae because this variant is difficult to detect on CPE screening media due to the low hydrolytic activity of this carbapenemase (8). OXA-244 is found mainly in Escherichia coli isolates (6). The blaOxA-244 gene is described in only 1 type of transposon, Tn51098, a 21.9-kb IS1R-based composite transposon

Author affiliations: INSERM, University Paris-Saclay,

Le Kremlin-Bicêtre, France (C. Emeraud, D. Girlich, R.A. Bonnin,

A.B. Joussett, T. Naas, L. Dortet); Bicêtre Hospital,

Le Kremlin-Bicêtre (C. Emeraud, A.B. Jousset, T. Naas, L. Dortet);

French National Reference Center for Antibiotic Resistance,

Le Kremlin-Bicêtre (C. Emeraud, R.A. Bonnin, A.B. Jousset,

T. Naas, L. Dortet)

DOI: https://doi.org/10.3201/eid2704.204459 that includes a truncated $\operatorname{Tn} 1999.2(\Delta \operatorname{Tn} 1999.2)$ and a fragment of the archetypal IncL $b l a_{\text {OXA-48 }}$-carrying plasmid, pOXA-48 (2).

Previous studies analyzed only a limited number of OXA-244-producing E. coli of an epidemic clone belonging to sequence type (ST) 38 that spread in countries in Europe $(3,5,7,9)$. More data on the epidemiology and genetics of OXA-244 are required to understand its spread in Europe. We used wholegenome sequencing (WGS) to characterize the epidemiology of OXA-244-producing E. coli circulating in France during 2016-2019.

\section{The Study}

During 2016-2019, the French National Reference Center identified 97 OXA-244-producing E. coli isolates. We performed WGS on all isolates by using the HiSeq (IIlumina Inc., https://www.illumina.com) sequencing platform (GenBank accession nos. in Appendix 1 Table, https://wwwnc.cdc.gov/EID/article/27/4/204459-App1.xlsx). We performed in silico multilocus sequence typing (MLST) by using the MLST 2.0 server (https://cge.cbs.dtu.dk/services/MLST). We identified 12 different sequence types (STs); the 5 most prevalent were ST38 ( $=37)$, ST361 ( $n=17)$, ST69 ( $n=$ 12), ST167 $(n=11)$, and ST10 $(n=8)$ (Figure 1). Among OXA-244-producing E. coli isolates, the prevalence of ST38 rose from 12\% in 2016 and to $47 \%$ in 2019.

On all 97 genomes of OXA-244-producing E. coli, we used a core genome single-nucleotide polymorphism (SNP)-based approach to create a phylogenetic tree by using CSIPhylogeny (https:/ / cge. cbs.dtu.dk/services/CSIPhylogeny). To identify clades within STs, we performed a nested phylogenetic analysis with isolates of each ST to construct a SNP matrix. Isolates within the same clade would be highly suggestive of patient-to-patient crosstransmission of the same strain. We considered 2 strains to be part of the same clade if they were separated by $<100$ SNPs along their common genome 


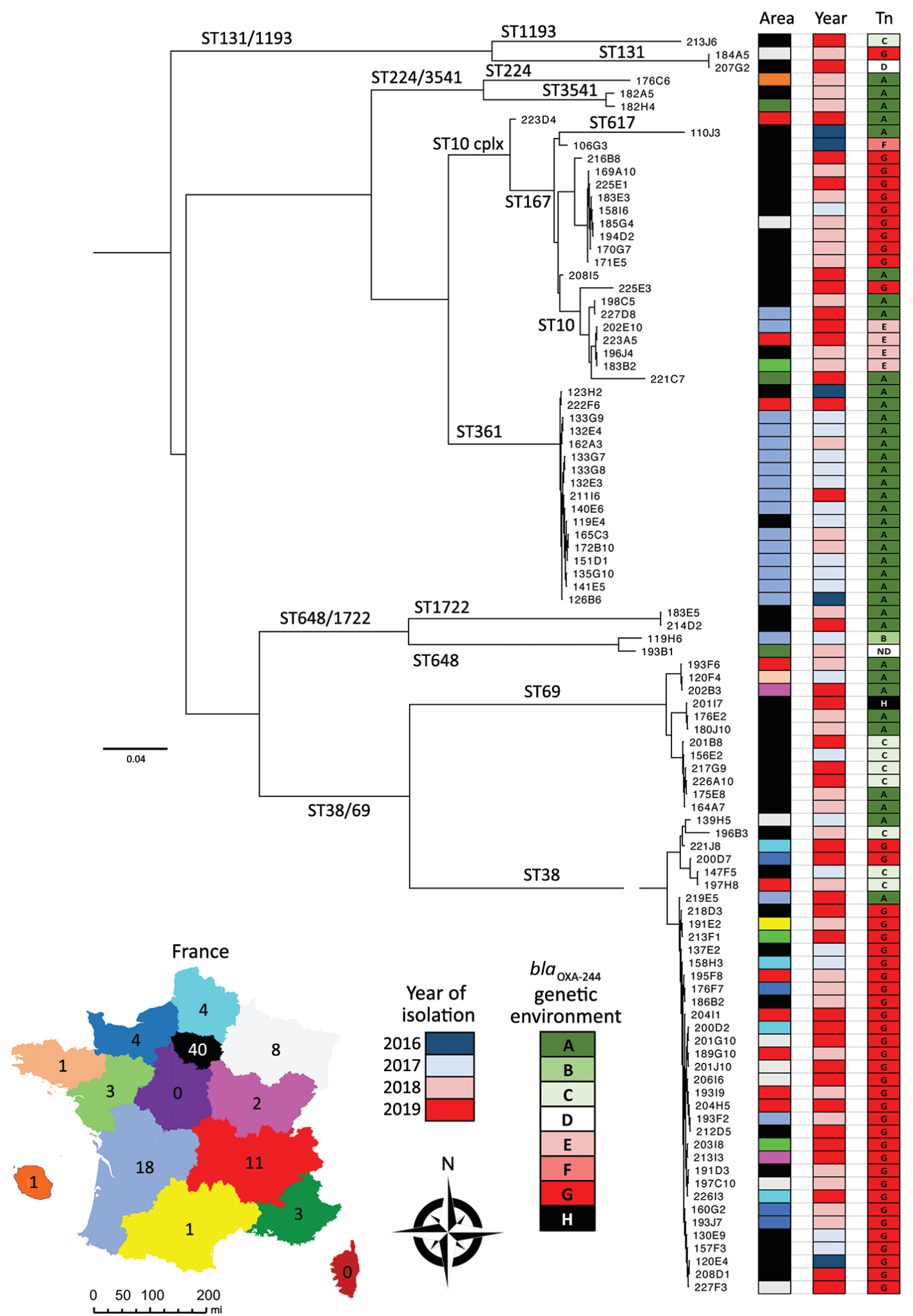

Figure 1. Phylogenetic relationship and geographic distribution of the 97 OXA-244-producing Escherichia coli isolates recovered in France, 2016-2019. Inset map shows regions of France; colors correspond to areas from which OXA-244-producing E. coli isolates were collected. The phylogenetic tree was constructed using CSIPhylogeny version 1.4 (https://cge.cbs.dtu.dk/services/CSIPhylogeny). Scale bar indicates nucleotide substitutions per site. ND, not detected; OXA, oxacillin; ST, sequence type. 
(Appendix 2 Figure, https://wwwnc.cdc.gov/ EID/article/27/4/20-4459-App2.pdf). We identified large clades corresponding to clonal dissemination of a single strain in the same area, including $5 / 12$ isolates of ST167 and 15/17 isolates of ST361. We identified $\leq 7$ different clades of ST38, and most (30/37) isolates belonged to the same clade (Appendix 2 Figure). However, these 30 ST38 isolates were collected in 9 different areas of France and 25 were isolated during 2018-2019 (Figure 1).

Because assembly of regions with repeated sequences was difficult with Illumina WGS data, we sequenced some isolates by using long read nanopore technology by using a Minlon (Oxford Nanopore, https://nanoporetech.com) sequencer (10). We performed WGS on 3 isolates belonging to the most prevalent STs: isolate

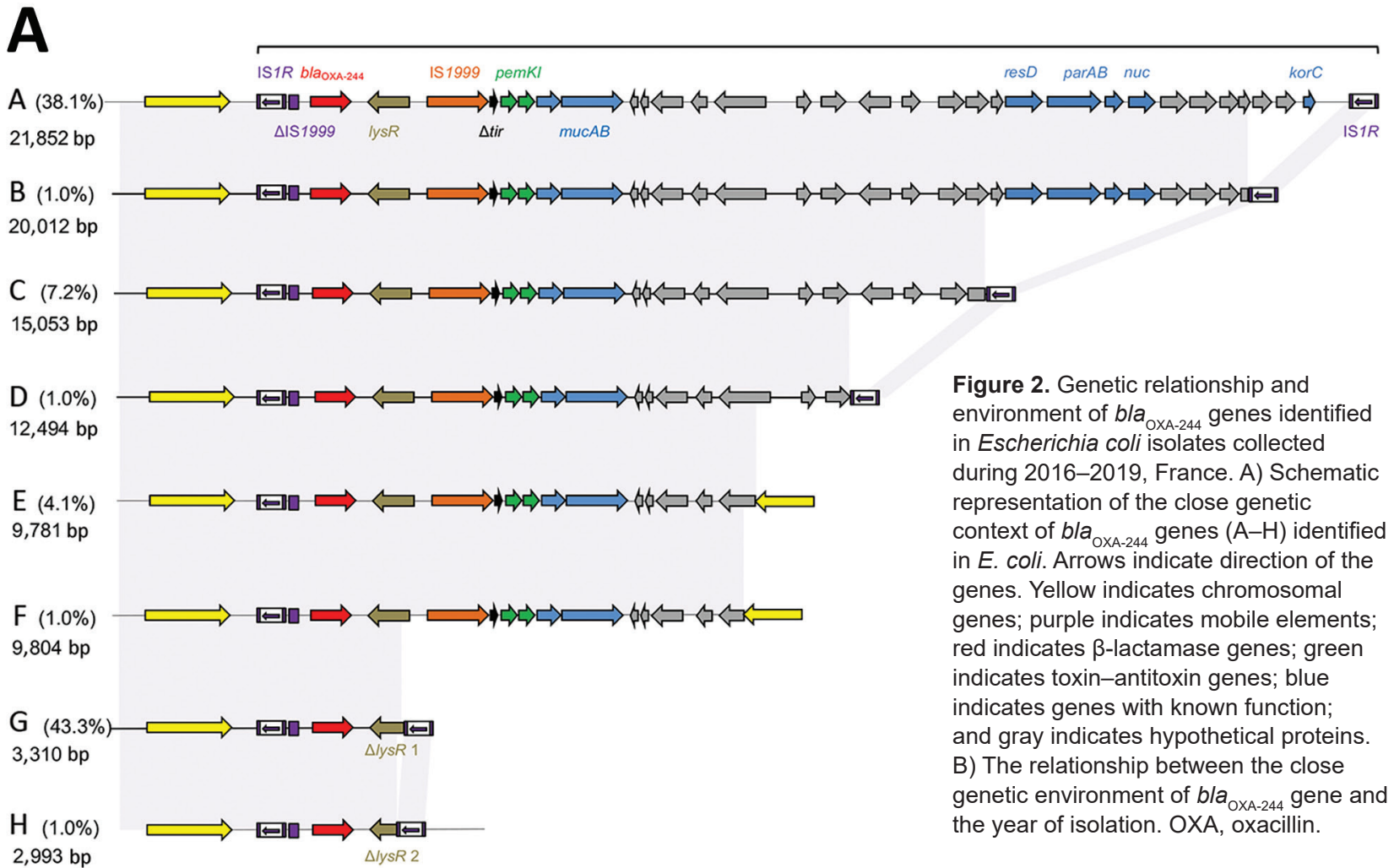

B

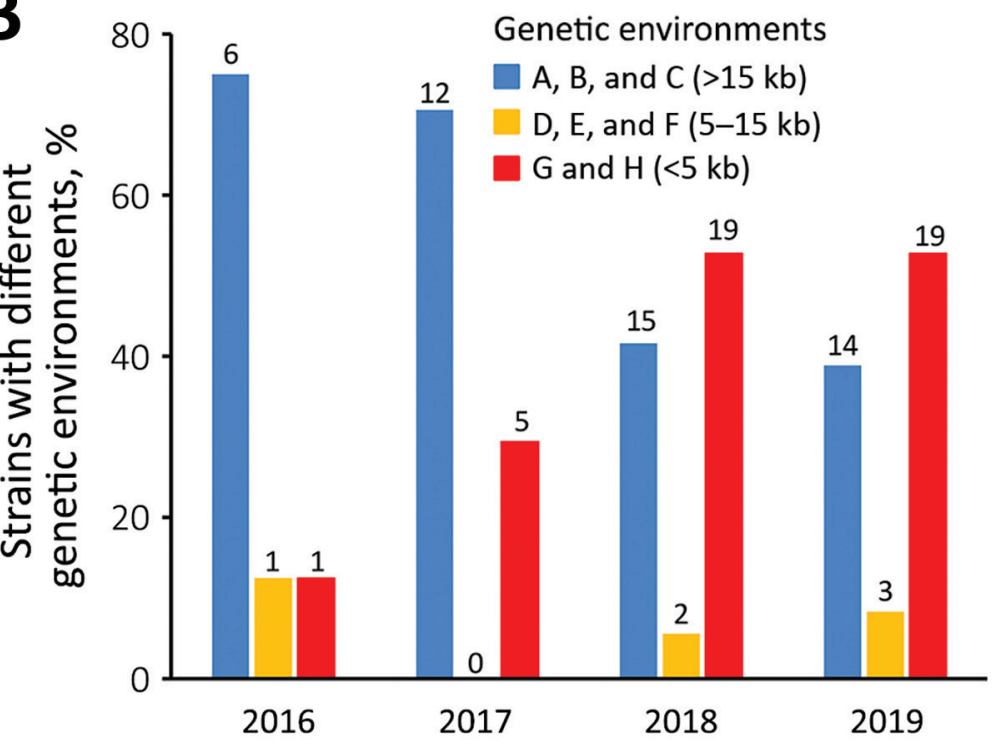


119E4 (ST361), isolate 120E4 (ST38), and isolate 156E2 (ST69) (Appendix 1 Table). We found a chromosomal localization of $b l a_{\mathrm{OXA}-244}$ gene in the 3 isolates. By combining data obtained by both WGS technologies, we reconstructed the different genetic environments of $b l a_{\text {OXA-244 }}$ gene and annotated the assembled sequences by using CLC Genomics Workbench version 12.0 software (QIAGEN, https://www.qiagen.com).

We detected 8 different genetic environments in our collection (Figure 2, panel A). Among the $97 \mathrm{E}$. coli isolates, 37 (38.1\%) possessed the $b l a_{\text {OXA-244 }}$ gene in Tn51098, a previously described transposon $(2,11)$ (Figure 2, panel A). Among the other 60 (61.9\%) isolates, we found $b l a_{\text {OXA-244 }}$ in the shorter form of Tn51098 (2,933$20,012 \mathrm{bp}$ ) (Figure 2, panel A). The bla ${ }_{\mathrm{OXA}-244}$ gene still was systematically included in a truncated Tn1999.2 ( $\Delta$ Tn1999.2), as described in E. coli VAL (2). For 44.3\% of isolates, the remnant Tn51098 was reduced in size (42 isolates with genetic environment $G$ and 1 with genetic environment $\mathrm{H}$ ) (Figure 2, panel A). We noted, the $b l a_{\mathrm{OXA}-244}$ gene was included in a $\Delta \operatorname{Tn} 1999.2$ where the lysR gene was truncated by the IS1R element. Of the 42 isolates sharing the genetic environment $G, 32$ (76.1\%) belonged to ST38. By separating the type of genetic environment according to the date of isolation, we noticed that the short forms were isolated during 2018-2019 (38/44 strains, 86\%) (Figure 2, panel B).

\section{Discussion}

Dissemination of ST38 OXA-244-producing E. coli has been observed in many countries in Europe (3-7) and a few other countries around the world $(12,13)$. However, most of these studies focused on ST38. Our results confirm the phenomenon of OXA-244-producing E. coli isolates in France because 38\% of isolates in our study belonged to ST38. In addition, we observed an increased number of ST38 isolates during 2018-2019. Phylogenetic analysis identified a substantial clade inside ST38 (Appendix 2 Figure), but massive dissemination of this clone in France likely does not correspond to cross transmission of a single strain in different areas. The few SNP differences identified among ST38 isolates suggest this clade emerged recently. Accordingly, inside this compact ST38, the $<100$ SNP cutoff used to discriminate between 2 clades might be lowered because it was recently described for another high-risk clone, Klebsiella pneumoniae carbapenemase-producing K. pneumoniae ST258 (14).

The other common STs noted in our study are ST361, ST167, ST69 and ST10. In Europe, OXA-244producing E. coli of ST69, ST167, and ST361 have been reported in Denmark (6), and ST69 and ST10 in Switzerland. Unlike what we observed with ST38, clones observed inside ST167 and ST361 mostly correlate with the same geographic area suggesting patient-topatient cross-transmission.

As previously described for ST38 E. coli VAL (2), we demonstrated the chromosomal location of the blaoxa-244 gene in 3 isolates belonging to the 3 main STs, ST38, ST361, and ST69. The chromosomal localization of bla ${ }_{\text {OXA-244 }}$ together with the intrinsic lower hydrolytic activity of OXA-244, compared with OXA-48, contribute to the difficulties in accurately detecting OXA-244-producing E. coli using classical screening media $(8,15)$, suggesting a large underestimation of the real spread of OXA-244 producers.

In 2013 , the $b l a_{\text {OXA-244 }}$ gene initially was reported to be embedded in a 21,852-bp transposon Tn51098, which contains $\Delta \operatorname{Tn} 1999.2(2)$. This structure still is present in $38.1 \%$ of OXA-244-producing E. coli. To our knowledge, Tn51098 is the sole genetic structure reported for $b l a_{\mathrm{OXA}-244}$. In our collection, $b l a_{\mathrm{OXA}-244}$ was embedded in truncated forms of Tn51098 in most isolates. Of note, in most (86.5\%) ST38 OXA-244-producing E. coli the close genetic context of the $b l a_{\text {OXA-244 }}$ gene was reduced to a small 3,310-bp fragment matching Tn51098 and corresponding to a truncated form of the Tn1999.2. In addition, the most recently collected isolates possess short versions of the Tn51098 compared with the isolates collected earlier (Figure 2, panel B). The effect on the clonal dissemination of this genome reduction around $b l a_{\mathrm{OXA}-244}$ gene (e.g., better fitness) remains undetermined. Further analysis on the $b l a_{\mathrm{OXA}-244}$ close genetic environment could elucidate the effects of this genome reduction.

\section{About the Author}

Dr. Emeraud is an assistant professor at the INSERM, Le Kremlin-Bicêtre, France. Her main research interests include epidemiology, genetics, and biochemistry of $\beta$-lactamases in gram negative bacteria.

\section{References}

1. Nordmann P, Dortet L, Poirel L. Carbapenem resistance in Enterobacteriaceae: here is the storm! Trends Mol Med. 2012;18:263-72. https://doi.org/10.1016/ j.molmed.2012.03.003

2. Potron A, Poirel L, Dortet L, Nordmann P. Characterisation of OXA-244, a chromosomally-encoded OXA-48-like $\beta$-lactamase from Escherichia coli. Int J Antimicrob Agents. 2016;47:102-3. https:// doi.org/10.1016/j.ijantimicag. 2015.10.015

3. Masseron A, Poirel L, Falgenhauer L, Imirzalioglu C, Kessler J, Chakraborty T, et al. Ongoing dissemination of OXA-244 carbapenemase-producing Escherichia coli in Switzerland and their detection. Diagn Microbiol Infect Dis. 2020;97:115059. https://doi.org/10.1016/j.diagmicrobio.2020.115059

4. European Centre for Disease Prevention and Control. Increase in OXA-244-producing Escherichia coli in the European Union/European Economic Area and the UK 
since 2013. Stockholm: the Centre; 2020 [cited 2020 Feb 18] https://www.ecdc.europa.eu/sites/default/ files / documents/RRA-E-coli-OXA-244-producing-E-coli-EU-EEAUK-since-2013.pdf.

5. Kremer K, Kramer R, Neumann B, Haller S, Pfennigwerth N, Werner G, et al. Rapid spread of OXA-244-producing Escherichia coli ST38 in Germany: insights from an integrated molecular surveillance approach; 2017 to January 2020. Euro Surveill. 2020;25:2000923. https:/ / doi.org/10.2807/ 1560-7917.ES.2020.25.25.2000923

6. Hammerum AM, Porsbo LJ, Hansen F, Roer L, Kaya H, Henius A, et al. Surveillance of OXA-244-producing Escherichia coli and epidemiologic investigation of cases, Denmark, January 2016 to August 2019. Euro Surveill. 2020;25. https://doi.org/10.2807/1560-7917.ES.2020.25.18.1900742

7. Falgenhauer L, Nordmann P, Imirzalioglu C, Yao Y, Falgenhauer J, Hauri AM, et al. Cross-border emergence of clonal lineages of ST38 Escherichia coli producing the OXA-48-like carbapenemase OXA-244 in Germany and Switzerland. Int J Antimicrob Agents. 2020;56:106157. https:// doi.org/10.1016/j.ijantimicag.2020.106157

8. Emeraud C, Biez L, Girlich D, Jousset AB, Naas T, Bonnin RA, et al. Screening of OXA-244 producers, a difficult-to-detect and emerging OXA-48 variant? J Antimicrob Chemother. 2020;75:2120-3.

9. Oteo J, Hernández JM, Espasa M, Fleites A, Sáez D, Bautista V, et al. Emergence of OXA-48-producing Klebsiella pneumoniae and the novel carbapenemases OXA-244 and OXA-245 in Spain. J Antimicrob Chemother. 2013;68:317-21. https://doi.org/10.1093/jac/dks383

10. Bonnin RA, Jousset AB, Gauthier L, Emeraud C, Girlich D, Sauvadet A, et al. First occurrence of the OXA-198 carbapenemase in Enterobacterales. Antimicrob Agents Chemother. 2020;64:e01471-19. https://doi.org/10.1128/ AAC.01471-19

11. Pitout JDD, Peirano G, Kock MM, Strydom K-A, Matsumura Y. The global ascendency of OXA-48-type carbapenemases. Clin Microbiol Rev. 2019;33:e00102-19. https:/ / doi.org/10.1128/CMR.00102-19

12. Abril D, Bustos Moya IG, Marquez-Ortiz RA, Josa Montero DF, Corredor Rozo ZL, Torres Molina I, et al. First report and comparative genomics analysis of a $b l a_{\text {OXA-244 }}$-harboring Escherichia coli isolate recovered in the American continent. Antibiotics (Basel). 2019;8:222. https://doi.org/10.3390/antibiotics8040222

13. Soliman AM, Ramadan H, Sadek M, Nariya H, Shimamoto T, Hiott LM, et al. Draft genome sequence of a bla $\mathrm{NDM}-1^{-}$and bla $_{\text {OXA-244 }}$-carrying multidrug-resistant Escherichia coli D-ST69 clinical isolate from Egypt. J Glob Antimicrob Resist. 2020;22:832-4. https://doi.org/10.1016/j.jgar.2020.07.015

14. David S, Reuter S, Harris SR, Glasner C, Feltwell T, Argimon S, et al.; EuSCAPE Working Group; ESGEM Study Group. Epidemic of carbapenem-resistant Klebsiella pneumoniae in Europe is driven by nosocomial spread. Nat Microbiol. 2019;4:1919-29. https://doi.org/10.1038/s41564-019-0492-8

15. Hoyos-Mallecot $Y$, Naas T, Bonnin RA, Patino R, Glaser P, Fortineau N, et al. OXA-244-producing Escherichia coli isolates, a challenge for clinical microbiology laboratories. Antimicrob Agents Chemother. 2017;61: e00818-17. https:// doi.org/10.1128/AAC.00818-17

Address for correspondence: Laurent Dortet, Service de Bactériologie-Hygiène, Hôpital de Bicêtre, 78 rue du Général Leclerc, 94275 Le Kremlin-Bicêtre CEDEX, France; email: laurent.dortet@aphp.fr

\section{EID SPOTLIGHT TOPIC}

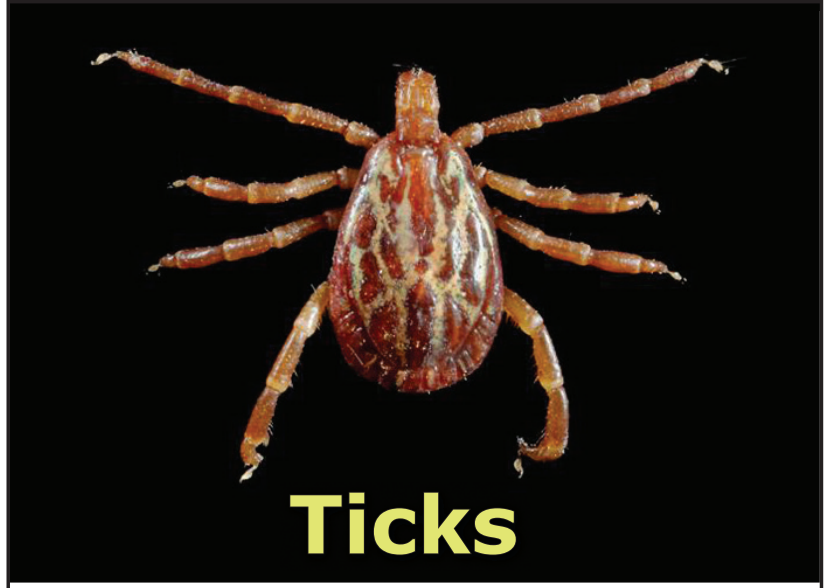

Ticks transmit a variety of different pathogens including bacteria, protozoa, and viruses which can produce serious and even fatal disease in humans and animals. Tens of thousands of cases of tickborne disease are reported each year, including Lyme disease. See the EID Lyme Disease Spotlight. Lyme disease is the most well-known tickborne disease. However, other tickborne illnesses such as Rocky Mountain spotted fever, tularemia, babesiosis, and ehrlichiosis also contribute to severe morbidity and more mortality each year.

Symptoms of tickborne disease are highly variable, but most include sudden onset of fever, headache, malaise, and sometimes rash. If left untreated, some of these diseases can be rapidly fatal.


\section{https://wwwnc.cdc.gov/eid/ page/tick-spotlight}

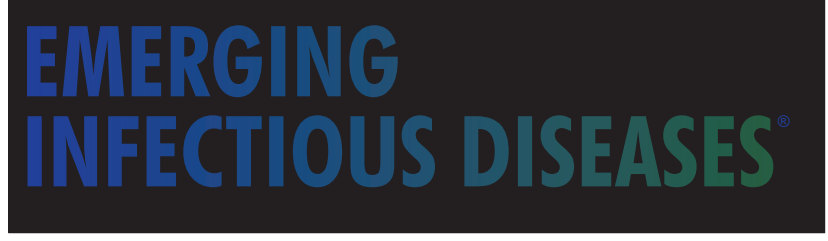

